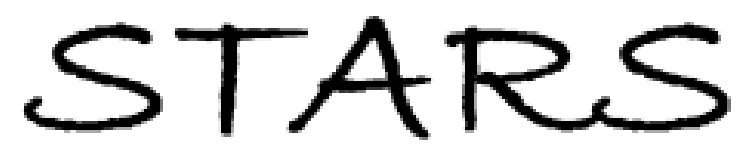

University of Central Florida

STARS

$1-1-2005$

\title{
Classification of imaging spectrometers for remote sensing applications
}

\author{
R. Glenn Sellar \\ University of Central Florida \\ Glenn D. Boreman \\ University of Central Florida
}

Find similar works at: https://stars.library.ucf.edu/facultybib2000

University of Central Florida Libraries http://library.ucf.edu

This Article is brought to you for free and open access by the Faculty Bibliography at STARS. It has been accepted for inclusion in Faculty Bibliography 2000 s by an authorized administrator of STARS. For more information, please contact STARS@ucf.edu.

\section{Recommended Citation}

Sellar, R. Glenn and Boreman, Glenn D., "Classification of imaging spectrometers for remote sensing applications" (2005). Faculty Bibliography 2000s. 5652.

https://stars.library.ucf.edu/facultybib2000/5652

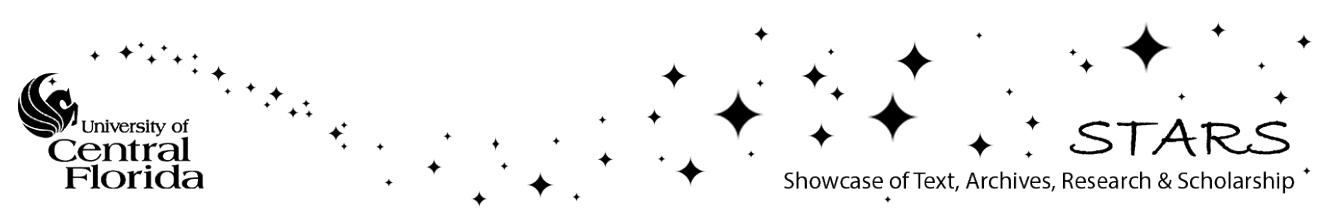




\section{Classification of imaging spectrometers for remote sensing applications}

\author{
R. Glenn Sellar,* MEMBER SPIE \\ University of Central Florida \\ Department of Physics \\ 12424 Research Parkway, Suite 400 \\ Orlando, Florida 32826
}

Glenn D. Boreman, FELLOW SPIE

University of Central Florida

CREOL/School of Optics

Orlando, Florida 32816-2700

\begin{abstract}
The continuing development of new and fundamentally different classes of imaging spectrometers has increased the complexity of the field of imaging spectrometry. The rapid pace at which new terminology is introduced to describe the new types of imaging spectrometers sometimes leads to confusion, particularly in discussions of the relative merits of the different types. In some cases, multiple different terms are commonly used to describe the same fundamental approach, and it is not always clear when these terms are synonymous. Other terminology in common use is overly broad. When a single term may encompass instruments that operate in fundamentally different ways, important distinctions may be obscured. In the interest of clarifying the terminology used in imaging spectrometry, we present a comprehensive system for classification of imaging spectrometers based on two fundamental properties: the method by which they scan the object spatially, and the method by which they obtain spectral information. (c) 2005 Society of PhotoOptical Instrumentation Engineers. [DOI: 10.1117/1.1813441]
\end{abstract}

Subject terms: imaging spectrometry, imaging spectrometer; hyperspectral imaging; hyperspectral imagery; hyperspectral imager; remote sensing.

Paper 040052 received Jan. 28, 2004; revised manuscript received Apr. 9, 2004; accepted for publication Apr. 29, 2004; published online Dec. 17, 2004.
Imaging spectrometers are designed to measure the energy or quanta collected from an object as a function of two spatial and one spectral dimension. The resulting 3-D dataset is often referred to as an object cube or data cube. To be truly an imaging spectrometer, the spatial sampling provided by the instrument must be essentially continuous; a multiobject spectrograph, which acquires spectra from a sparse array of positions across the field of the object, is not an imaging spectrometer in the strict definition of the term, since it cannot provide what would generally be considered images. If an absolute radiometric calibration is also provided, then the instrument is an imaging spectroradiometer, though this term is seldom used.

Imaging spectrometers used for remote sensing may be divided into classes based on two aspects: the method by which they achieve spatial discrimination, and the method by which they achieve spectral discrimination. Methods of acquiring spatial information include whiskbroom, pushbroom, framing, and the relatively new class we refer to as windowing. In our taxonomy, a whiskbroom-scanning instrument employs a zero-dimensional or spot or point fieldof-view (FOV), which scans the object in both the alongtrack and cross-track directions, while a pushbroomscanning instrument employs a 1-D FOV, which is scanned in only one direction. Typically the scan direction for a pushbroom-scanning instrument is the along-track direction, but there are examples of pushbroom instruments that scan in the cross-track direction. ${ }^{1}$ While the latter have sometimes been referred to as whiskbroom rather than

\footnotetext{
*Current affiliation: Jet Propulsion Laboratory, California Institute of Technology, Pasadena, CA 91109. Electronic mail: glenn.sellar@jpl.nasa.gov

0091-3286/2005/\$22.00 @ 2005 SPIE
}

pushbroom, the distinction between a zero-dimensional FOV scanned in two directions versus a 1-D FOV scanned in one direction is the more fundamental distinction, so we classify all imaging spectrometers with a 1-D FOV as pushbroom instruments, regardless of the direction of the scanning motion. It should also be noted that it is the shape of the FOV that determines the spatial discrimination class; in most pushbroom instruments this restriction of the FOV is obtained by placing a slit aperture in an intermediate focal plane, but any other method of restricting the FOV to be essentially 1-D will put the instrument in the pushbroom class.

A framing instrument employs a 2-D FOV, which remains fixed on the object during acquisition, though multiple acquisitions may be required to cover an object of general length in the along-track direction. We use "framing" as synonymous to "staring," but prefer the former term since if the scene to be observed is longer than the length of a single FOV, then multiple frames must be acquired and thus framing seems a clearer description. We use the term windowing to describe the relatively new class of instruments that employ a 2-D FOV that moves across the object in a continuous fashion in the along-track direction. Windowing is actually distinct from the time-delay integration technique used by some panchromatic imagers, since in a windowing instrument a distinct exposure is acquired each time the FOV moves forward by one ground sample and no integration occurs.

Methods of acquiring spectral information include the familiar filtering, dispersive, and interferometric techniques. Dispersive instruments may use either a prism or grating. We define the interferometric class as Fouriertransform spectrometers (FTS), which are based on twobeam interferometers such as the Michelson, Mach-Zender, 
Table 1 Classification of imaging spectrometers.

\begin{tabular}{|c|c|c|c|c|}
\hline \multirow[b]{3}{*}{ Spectral } & \multicolumn{4}{|c|}{ Along-track scanning } \\
\hline & Whiskbroom & Pushbroom & Windowing & Framing \\
\hline & 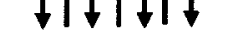 & U & & \\
\hline Filtering & $\begin{array}{l}\text { - Multiband } \\
\text { radiometer }\end{array}$ & $\begin{array}{l}\text { - (No known } \\
\text { examples) }\end{array}$ & $\begin{array}{l}\text { - Filter array } \\
\text { - Wedge filter } \\
\text { - Linear variable } \\
\text { filter }\end{array}$ & $\begin{array}{l}\text { - Band-sequential } \\
\text { - Filter wheel } \\
\text { - Tunable filter } \\
\text { (AOTF or LCTF) }\end{array}$ \\
\hline Dispersive & - Grating or prism & - Grating or prism & $\begin{array}{c}\cdot \text { (No known } \\
\text { examples) }\end{array}$ & $\begin{array}{l}\text { - Image slicer } \\
\text { - Tomographic }\end{array}$ \\
\hline Interferometric & $\begin{array}{l}\text { - Traditional FTS } \\
\text { - Point FTS } \\
\text { (Michelson) }\end{array}$ & $\begin{array}{c}\text { - Static FTS } \\
\text { (Sagnac) }\end{array}$ & $\begin{array}{l}\text { - Static FTS } \\
\text { (Mach-Zender, } \\
\text { Sagnac) }\end{array}$ & $\begin{array}{l}\text { - Traditional FTS } \\
\text { (Michelson) }\end{array}$ \\
\hline
\end{tabular}

or Sagnac. Multiple-beam interferometers such as the Fabry-Perot belong more appropriately to the filtering class, as do interference filters, since they obtain spectral discrimination by passing at any instant only a narrow range of wavelengths, while blocking all other wavelengths.

This classification scheme, with examples of commonly used terms for each class, is illustrated in Table 1. Although there are 12 possible classes in this scheme, we are only aware of existing instruments in ten of these classes. Some of these classes are well established and are likely to be familiar: instruments in the filtering whiskbroom class are often known as multiband radiometers, the filtering framing class includes filter-wheel-equipped cameras, instruments in the dispersive pushbroom class are often referred to as prism (imaging) spectrometers or grating (imaging) spectrometers, and the interferometric framing class includes the traditional imaging FTS using a Michelson interferometer. Other classes are relatively novel additions to the field. The interferometric pushbroom class ${ }^{2,3}$ uses static or spatially modulated interferometers such as the Sagnac interferometer. The interferometric windowing class ${ }^{4,5}$ also uses a static interferometer but, unlike the interferometric pushbroom spectrometer, does not require a narrow field mask (or slit). The filtering windowing class ${ }^{6,7}$ places in an image plane a filter designed with a passband that varies with position. The dispersive framing class includes both instruments that use an image slicer to rearrange the elements of a 2-D FOV into a 1-D form at the entrance slit of a prism or grating spectrometer, ${ }^{8}$ as well as instruments that use crossed gratings to produce multiple projections of the object cube from which the elements of the object cube are estimated using tomographic algorithms. ${ }^{9}$

The classification scheme serves to group instruments according their fundamental principles of operation, rather than by the specific devices employed in the design. For example, instruments in the dispersive class may use either a prism or grating. They are grouped in the same spectral class because both devices provide fundamentally the same function to the instrument: that of sorting rays by wavelength into different angles of propagation. Similarly, instruments using a filter wheel, an acousto-optical tunable filter, or a liquid-crystal tunable filter all belong to the same fundamental class: filtering framing. The term "band sequential" has been aptly used for this class, but the latter term does not indicate the relationship of this class to other types of imaging spectrometers. The term "filtering framing," however, indicates that this class shares characteristics in common with related classes. In common with the related filtering windowing class, the filtering framing class selects only a single spectral band to reach a detector at a particular instant, and in common with an interferometric framing instrument, a filtering framing instrument also has a 2-D FOV that remains stationary on the object during acquisition.

The matrix character of this classification scheme helps to clarify the principles of operation of novel instruments by analogy with more familiar instruments. For example, the interferometric windowing class obtains spatial discrimination in the same way the filtering windowing class does, while obtaining spectral discrimination in the same way as the interferometric framing class. Both filtering windowing and interferometric windowing instruments use a 2-D FOV that sweeps across the object (typically through the motion of an aircraft or spacecraft platform). In a filtering windowing instrument, the detected wavelength varies as a function of position in the FOV, while in an interferometric windowing instrument, it is path difference that varies with position in the FOV. In the interferometric windowing class, the spectrum is obtained by applying a Fourier transform to the variation of collected energy with path difference, in the same fashion employed by the more familiar interferometric framing class.

This matrix classification approach also helps to distinguish between instruments that use the same devices but operate in fundamentally different ways. For example, Sagnac interferometers are used in both interferometric pushbroom instruments and interferometric windowing instruments, though these two classes obtain spatial information in fundamentally different ways. Michelson interferometers are typically used in interferometric framing instruments, while a Mach-Zender interferometer was used for the first interferometric windowing instrument. ${ }^{4}$ In principle, however, any of these three interferometers may be used for any of the four interferometric classes. Classifying 
instruments by how they obtain spatial and spectral discrimination, as we have done here, rather than by the specific devices (i.e., the type of interferometer, type of filter, or type of disperser), provides a more fundamental and useful designation.

\section{Acknowledgments}

This research was funded by NASA under grant number NAG5-10730.

\section{References}

1. C. G. Simi, E. M. Winter, M. M. Williams, and D. C. Driscoll, "Compact airborne spectral sensor (COMPASS)," Proc. SPIE 4381, 129136 (2001).

2. W. H. Smith and W. V. Schempp, "Digital array scanned interferometers for astronomy," Exp. Astron. 1, 388-405 (1991).

3. L. J. Otten, J. B. Rafert, A. Meigs, B. Jones, R. Prinzing, R. Fronterhouse, R. G. Sellar, and P. Hodge, "The engineering model for the MightySat II.1 hyperspectral imager," Proc. SPIE 3221, 412-420 (1997).

4. R. F. Horton, "Optical design for a high-etendue imaging Fouriertransform spectrometer" Proc. SPIE 2819, 300-315 (1996).

5. W. J. Slough, J. B. Rafert, C. A. Rohde, and C. L. Hart, "THRIFTI Tomographic hyperspectral remote imaging Fourier transform interferometer," Proc. SPIE 3393, 207-216 (1998).

6. J. C. Demro, R. Hartshorne, and L. M. Woody, "Design of a multispectral, wedge filter, remote sensing instrument incorporating a multi-port, thinned, CCD area array," Proc. SPIE 2480, 280-294 (1995).

7. D. C. Reuter, D. E. Jennings, G. H. McCabe, J. W. Travis, V. T. Bly, A. T. La, T. L. Nguyen, M. D. Jhabyala, P. K. Shu, and R. D. Endres, "Hyperspectral sensing using the linear etalon imaging spectral array," Proc. SPIE 2957, 154-161 (1997).

8. E. H. Richardson, J. M. Fletcher, and W. A. Grundman, "Image slicers," Proc. of IAU Colloquium 79, 469 (1984).

9. C. E. Volin, T. M. Gleeson, M. R. Descour, and E. L. Dereniak, "Portable computed-tomography imaging spectrometer," Proc. SPIE 2819, 224-230 (1996).

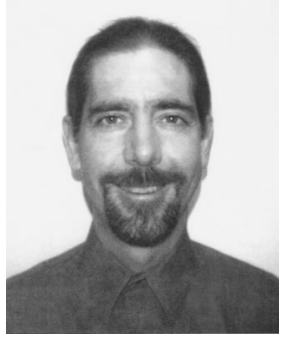

R. Glenn Sellar received a BEng degree in engineering physics from McMaster University in 1986, a MS degree in space sciences from the Florida Institute of Technology in 1993, and a PhD in optics from the University of Central Florida in 2003. He has 16 years of experience in the design, integration, and testing of imaging spectrometers, for ground-based, airborne, and spaceborne applications. He worked as an optical engineer on the Wind Imaging Interferometer (WINDII) for the Upper Atmosphere Research Satellite (UARS) and designed the optical system for the Fourier Transform HyperSpectral Imager (FTHSI) on MightySat II. He is member of SPIE and is currently developing imaging spectrometers for planetary science and earth science applications at the Jet Propulsion Laboratory.

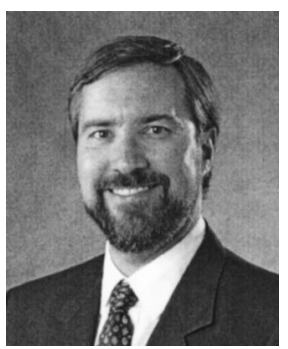

Glenn D. Boreman received a BS in optics from the Institute of Optics, University of Rochester and a PhD in optics from the Optical Sciences Center, University of Arizona. Since 1984, he has been on the faculty of the University of Central Florida, where he is presently Trustee Chair Professor of Optics. He currently serves as the Editor-in-Chief of Applied Optics, and is a past member of the SPIE Board of Directors. He is co-author of Infrared Detectors and Systems (Wiley), author of Basic Electro-Optics for Electrical Engineers, and Modulation Transfer Function in Optical and ElectroOptical Systems, and editor of Infrared Technology 1988-1998 (all SPIE Press). He is a fellow of OSA and SPIE. Along with his students, he received the 1995 Kingslake Medal from SPIE. 